\title{
Symmetric Division and Expression of Its Regulatory Gene Numb in Human Cervical Squamous Carcinoma Cells
}

\author{
Huaizeng Chen $^{\mathrm{a}}$ Xiaojun Chen ${ }^{\mathrm{b}}$ Feng Ye $\mathrm{e}^{\mathrm{a}, \mathrm{b}}$ Weiguo Lu $\mathrm{u}^{\mathrm{a}, \mathrm{b}}$ Xing Xie $\mathrm{X}^{\mathrm{a}, \mathrm{b}}$ \\ aWomen's Reproductive Health Laboratory of Zhejiang Province, Women's Hospital, School of Medicine, \\ ${ }^{b}$ Department of Gynecologic Oncology, Women's Hospital, School of Medicine, Zhejiang University, \\ Hangzhou, China
}

\section{Key Words}

Cell division $\cdot$ Numb $\cdot$ Cervical carcinoma

\begin{abstract}
Objective: To investigate the mode of cell division and Numb expression in cervical squamous cells. Method: We measured the cleavage angles of cervical squamous epithelia basal cells and Numb protein expression in 50 cases of cervical squamous cell carcinomas (CSCC), 59 cervical intraepithelial neoplasias (CIN) grade II-III, 21 CIN grade I and 48 controls. Results: We found an increase in horizontal divisions in CIN grade I and a decrease in horizontal divisions in CIN grade II-III. Numb expression had a tendency to be stronger from control to CSCC. In CSCC there was a significantly higher expression of mRNA for the Numb-PRR ${ }^{\mathrm{L}}$ isoform but not for the Numb-PRR ${ }^{S}$ isoform. Conclusion: Our findings suggest that in the initial stage of CSCC, carcinomatous cells may acquire an increase of symmetric division by altering cleavage orientation and proliferation. Expression of Numb protein in Cervical squamous epithelia and an increased expression of Numb-PRR ${ }^{L}$ isoforms may be among the mechanisms involved in the genesis of CSCC.
\end{abstract}

Copyright $\odot 2009$ S. Karger AG, Basel

\section{Introduction}

There are 2 cell division modes: asymmetric division and symmetric division [1-3]. Asymmetric division is the primary mechanism for generating cell fate diversity because it always produces 2 distinct daughter cells: 1 remains as the stem or progenitor cell that reenters the cell cycle and the other goes into cellular differentiation. On the other hand, symmetric division is believed to be associated with cellular proliferation because it usually produces 2 identical daughter cells, which can reenter the cell cycle after mitosis and serve to expand the pool of progenitor cells [4]. So we presume that malignant cells may possess uncontrolled proliferating ability through repeated symmetric divisions, and alterations of regulatory molecules of cell division may play roles in the process of malignant cell division.

In 1995, Chenn and McConnell [5] found that different cell cleavage orientations were consistent with different cell division modes in neuroepithelial cells. A cell cleavage orientation with its mitotic spindle aligned horizontally to the plane of the neuroepithelium (which we classified as 'horizontal' division) represents symmetric cell division. By contrast, a cell cleavage orientation with its mitotic spindle aligned vertically to the plane of the neuroepithelium (which we classified as 'vertical' division) represents

Women's Reproductive Health Laboratory of Zhejiang Province

Department of Gynecologic Oncology, Women's Hospital, School of Medicine Zhejiang University, Xueshi Rd 2, Hangzhou 310006 (China)

Tel. +86 5718706 1501, Fax +86 5718706 1878, E-Mail whxiexing@163.com 
asymmetric cell division. Recent studies have further verified that the cells are able to change the division mode through altering their cleavage orientation [6].

Whether cell-fate determinants with a polarized intracellular distribution along the apical-basal axis of the neuroepithelial cells are equally distributed into the daughter cells or not has been regarded as determining whether the cell division mode is symmetric or asymmetric [6-8]. Studies found that Numb is one of the very important cell fate determinants [8-10]. It is a plasma membrane-associated cytoplasmic protein, and contains 2 protein-protein interaction domains, a phosphotyrosinebinding domain and a proline-rich region (PRR) that functions as an SH3-binding domain $[11,12]$. Four Numb isoforms have been identified. Distinct human NumbPRR isoforms play different roles in differentiation and proliferation. Numb-PRR ${ }^{\mathrm{L}}$ isoforms promote cell proliferation, whereas Numb-PRR ${ }^{S}$ isoform enhances differentiation [13].

In the present study, we measured cell division mode and Numb expression in the cervical squamous cell carcinomas and its precursor lesions, to verify the association of cell division mode with the development of cervical carcinoma.

\section{Materials and Methods}

\section{Tissue Samples}

Tissue samples were obtained from the Women's Hospital, School of Medicine, Zhejiang University between 2002 and 2005. Tissue specimens were divided into 3 groups: cervical intraepithelial neoplasia grade I (CIN1), grade II-III (CIN2/3; containing CIN2, CIN3 and carcinoma in situ) and invasive cervical squamous cell carcinomas (CSCC). Cervical normal squamous epithelium was obtained from the patients who underwent hysterectomy due to diseases other than cancer. No CSCC patient received any chemotherapy or radiotherapy. Informed consent was obtained from each patient. This study was approved by the hospital ethics committee.

We studied a total of 178 paraffin-embedded cervical tissue sections: 50 cases of CSCC, 59 CIN2/3, 21 CIN1 and 48 cervical normal squamous epithelia. Cleavage angles of basal cells in mitoses were measured in CIN and normal cervical epithelia, and Numb expression was detected in all the cases. In addition, 65 fresh frozen tissue samples, including 37 cases of CSCC and 28 noncancerous cervical controls, were used for Numb-PRR mRNA splicing isoform analysis.

\section{Measurement of Cell Cleavage Angles}

The basal cells during metaphase, anaphase and early telophase of mitosis were selected as objects. A tangent line was made through a point where the basal cell in mitosis touched the basement membrane. The cell cleavage angle was measured between the central axis of the cleavage plane and the tangent line $[5,6]$. The cell cleavage angle was not measured in CSCC because it was not possible to identify the basement membrane.

Immunohistochemistry

The sections were incubated with primary antibody (rabbit anti-Numb polyclonal antibody, 1:40; Upstate), and was visualized using an HRP-conjugated polymer detection system (Zymed). All slides were counterstained with hematoxylin. The negative control was subjected to the same technique except that the primary antibody was replaced by phosphate-buffered saline. The specificity of the anti-Numb polyclonal antibody was checked using Western blotting and only 2 bands were visualized, corresponding to the Numb-PRR ${ }^{\mathrm{L}}$ and Numb-PRR $\mathrm{S}$.

Immunohistochemical results were evaluated as follows: negative (-) for no detectable staining, (+) for weak but definitely detectable staining, $(++)$ for moderate staining, $(+++)$ abundant staining. All the evaluations were made by 2 independent pathologists.

Reverse Transcription-Polymerase Chain Reaction (RT-PCR)

Total RNA was extracted by Trizol Reagent (Invitrogen) according to the manufacturer's instructions. $5 \mu \mathrm{g}$ of total RNA was reverse transcribed using a M-MuLV cDNA synthesis system (MBI). The PRR isoforms were: sense 5'-CGATGACCAAACCAGTGACAG-3', antisense 5'-AGAGGGAGTACGTCTATGACCG-3' (125-bp and/or 269-bp products) [10]. $\beta$-actin primers were: sense $5^{\prime}$-TTCCAGCCTTCCTTCCTGG-3', antisense $5^{\prime}$ TTGCGCTCAGGAGGAGCAAT-3' (224-bp product). NumbPRR isoform fragments were amplified through 30 cycles, and $\beta$-actin through 28 cycles, using the following amplification profiles: $94^{\circ} \mathrm{C}$ for $45 \mathrm{~s}, 60^{\circ} \mathrm{C}$ for $45 \mathrm{~s}, 72^{\circ} \mathrm{C}$ for $45 \mathrm{~s}$ (Numb-PRR isoforms) or $94^{\circ} \mathrm{C}$ for $30 \mathrm{~s}, 64^{\circ} \mathrm{C}$ for $30 \mathrm{~s}, 72^{\circ} \mathrm{C}$ for $30 \mathrm{~s}$ ( $\beta$-actin).

PCR products were visualized by $1.2 \%$ agarose electrophoresis. The Numb-PRR isoform expression was standardized with $\beta$-actin. These RT-PCR assays were performed independently 3 times.

\section{Statistical Analysis}

Statistical analysis was carried out using SPSS version 12.0. Cell cleavage angles were evaluated with the $\chi^{2}$ test. Immunochemical staining of Numb was analyzed using the Kruskal-Wallis test. Semiquantative RT-PCR analysis of Numb-PRR mRNA was compared by unpaired Student's t tests. Differences were considered to be statistically significant at $\mathrm{p}<0.05$, bilaterally.

\section{Results}

\section{Cell Cleavage Angles}

Cleavage angles of 340 mitotic basal cells were measured (fig. 1): 170 CIN2/3, 67 CIN1 and 103 normal controls. The distribution of the cleavage angles were significantly different among CIN2/3, CIN1, and control. In CIN $2 / 3$, the $0^{\circ}$ to $30^{\circ}$ division was significantly increased (table 1). 
Fig. 1. White arrows show the basal cell in mitosis. a Controls. b CIN1. c CIN2/3. d CIN2/3. HE stain. $\times 400$.
Fig. 2. Immunostaining of Numb. a Control. b CIN1. c CIN2/3. d CSCC. $\times 400$. The distinct brown coloration is located mainly in the cytoplasm of positive cells.
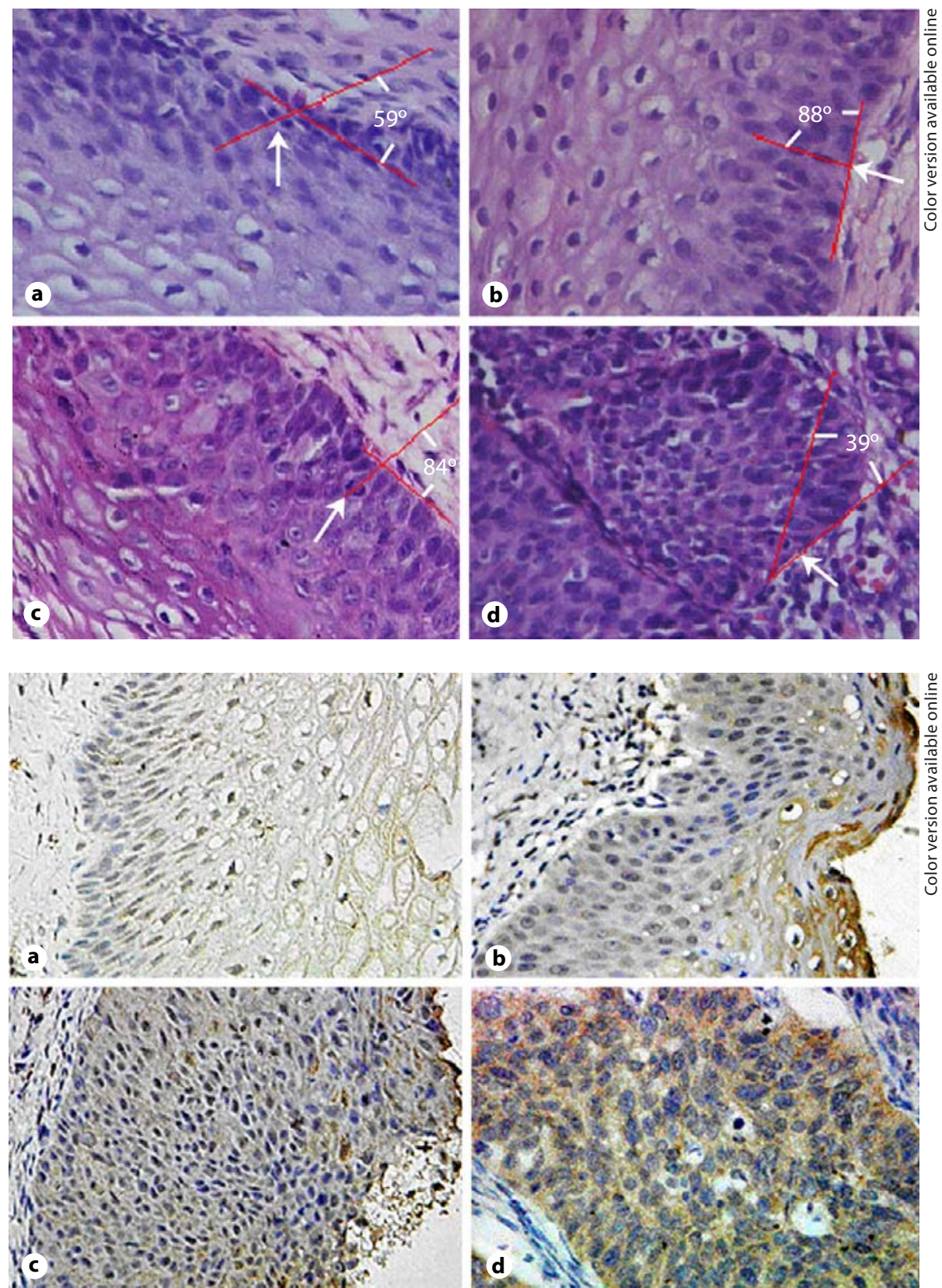

\section{Expression of Numb Protein}

Expression of Numb was found in squamous epithelial cells in all of the CSCC, CIN2/3, CIN1 and controls. Numb expression appeared to be diffused and had no asymmetric localization (fig. 2). The Numb expression gradually became stronger from normal control through to CSCC, except there was no significant difference between CIN1 and control (table 2).

Symmetric Division and Numb
Expression of Numb Isform mRNA

64 cases including CSCC and noncancerous controls expressed Numb-PRR ${ }^{\mathrm{L}}$ and Numb-PRR ${ }^{\mathrm{S}}$ mRNA (fig. 3a), while 1 control case only expressed Numb$\mathrm{PRR}^{S}$ mRNA. Semiquantitative analysis of expression of Numb-PRR isoform mRNA was made through normalized $\beta$-actin (table 3). Numb-PRR ${ }^{\mathrm{L}}$ mRNA expression was significantly higher in CSCC than that in noncancerous cervical controls (fig. $3 \mathrm{~b}$ ), but Numb-PRR ${ }^{S}$

Pathobiology 2009;76:149-154 

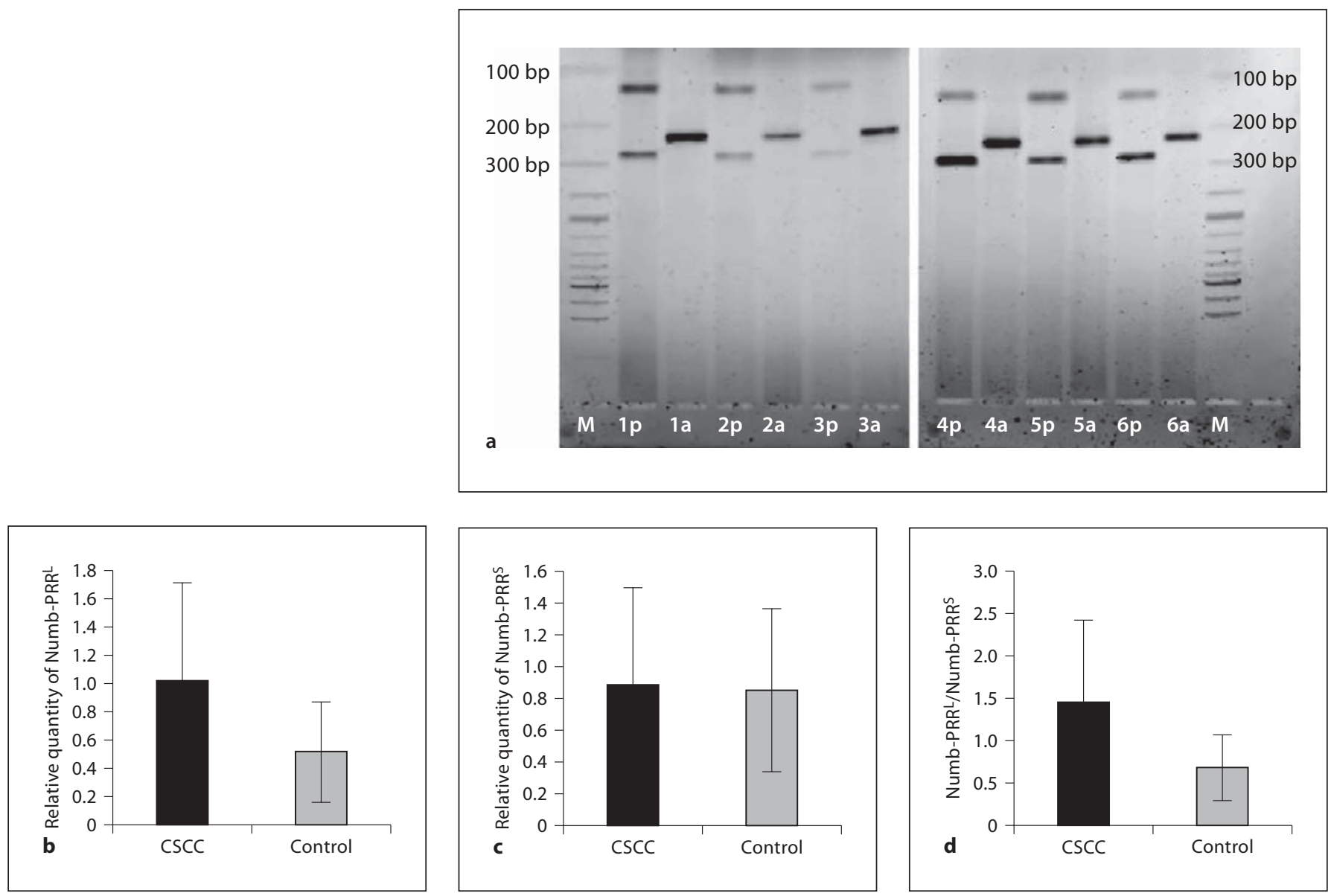

Fig. 3. a Expression of Numb-PRR isoform mRNA (Numb-PRR 269 bp; Numb-PRR ${ }^{S} 125$ bp). $p=$ Numb-PRR isoforms; $\mathrm{a}=\beta$-actin; $1-3=$ control group; $4-6=$ CSCC group. b Expression of Numb-PRR ${ }^{\mathrm{L}} \mathrm{mRNA}$. c Expression of Numb-PRR mRNA. d Expression ratio between Numb-PRR ${ }^{\mathrm{L}}$ and Numb-PRR ${ }^{\mathrm{S}}$.

Table 1. Cleavage angles of basal cells

\begin{tabular}{lcll}
\hline Cleavage angles & Control, n (\%) & CIN1, n (\%) & CIN2/3, n (\%) \\
\hline $0^{\circ}$ to $30^{\circ}$ & $56(54.4)$ & $27(40.3)$ & $92(54.1)$ \\
$30^{\circ}$ to $60^{\circ}$ & $20(19.4)$ & $18(26.9)$ & $54(34.8)$ \\
$60^{\circ}$ to $90^{\circ}$ & $27(26.2)$ & $22(32.8)$ & $24(14.1)$ \\
Total & $103(100)$ & $67(100)$ & $170(100)$ \\
\hline
\end{tabular}

Comparison between all 3 groups: $\chi^{2}=15.030, \mathrm{p}=0.005$ CIN2/3 vs. control: $\chi^{2}=8.632, \mathrm{p}=0.013$. CIN2/3 vs. CIN1: $\chi^{2}=$ $10.883, \mathrm{p}=0.004$. CIN1 vs. control: $\chi^{2}=3.271, \mathrm{p}=0.195$.
Table 2. Numb protein expressions

\begin{tabular}{lrrrr}
\hline \multirow{2}{*}{ Staining intensity } & \multicolumn{4}{c}{ Percentage of cases expressing Numb } \\
\cline { 2 - 5 } & control & CIN1 & CIN2/3 & CSCC \\
\hline+++ & 4.2 & 4.8 & 18.6 & 30.0 \\
++ & 31.3 & 33.3 & 61.0 & 64.0 \\
+ & 64.5 & 61.9 & 20.4 & 6.0 \\
- & 0.0 & 0.0 & 0.0 & 0.0 \\
Total & 100.0 & 100.0 & 100.0 & 100.0 \\
\hline
\end{tabular}

Comparison between all 4 groups: $\mathrm{H}=49.543$, $\mathrm{p}<0.001$. CSCC vs. $\mathrm{CIN} 2 / 3$ : $\mathrm{H}=4.657, \mathrm{p}=0.031$. CSCC vs. $\mathrm{CIN} 1: \mathrm{H}=21.300, \mathrm{p}<$ 0.001. CSCC vs. control: $\mathrm{H}=36.787, \mathrm{p}<0.001$. CIN2/3 vs. CIN1: $\mathrm{H}=11.395, \mathrm{p}=0.001$. CIN2/3 vs. control: $\mathrm{H}=21.537, \mathrm{p}<0.001$. CIN1 vs. control: $\mathrm{H}=0.0470, \mathrm{p}=0.828$. 
Table 3. Semiquantative analysis of Numb-PRR isoforms mRNA ( $\mathrm{x} \pm \mathrm{s})$

\begin{tabular}{lllll}
\hline Groups & $\mathrm{n}$ & Numb-PRR $^{\mathrm{L}}$ & Numb-PRR $^{\mathrm{S}}$ & $\begin{array}{l}\text { Numb-PRR } \\
\text { Numb-PRR }\end{array}$ \\
\hline Control & 28 & $0.515 \pm 0.354$ & $0.854 \pm 0.515$ & $0.685 \pm 0.380$ \\
CSCC & 37 & $1.026 \pm 0.683^{\mathrm{a}}$ & $0.888 \pm 0.610^{\mathrm{b}}$ & $1.453 \pm 0.981^{\mathrm{a}}$ \\
\hline
\end{tabular}

${ }^{\mathrm{a}} \mathrm{p}<0.001 ;{ }^{\mathrm{b}} \mathrm{p}=0.812$ vs. control groups

mRNA expression was not different between 2 groups (fig. 3c). Furthermore, the ratio between Numb-PRR ${ }^{\mathrm{L}}$ and Numb-PRR ${ }^{S}$ expression was also significantly higher in CSCC compared with noncancerous cervical controls (fig. 3d).

\section{Discussion}

It has been found that different cell cleavage orientations lead to distinct cell division modes. Horizontal division represents a symmetric cell division, while vertical division represents an asymmetric cell division $[5,6]$. This finding provides us with a simple morphological method for determining cell division modes.

A recent study on embryonic epidermal development showed that most of the epidermis is single-layered at early embryonic stages, with cell divisions occurring horizontally and representing mainly proliferation. However, the situation changes and most cell divisions became vertical and differentiated at the later stages when epidermal stratification forms [14]. Such results suggest that cell division modes, including symmetric and asymmetric, may be a key mechanism in regulating cell proliferation and differentiation.

In the present study, the results showed an increase in horizontal divisions as well as a decrease in vertical divisions in CIN1 when compared with normal squamous epithelium, although the difference was not significant. Unexpectedly, horizontal divisions were reduced, but oblique divisions and vertical divisions were found to rise again in CIN2/3 compared with CIN1 and controls. Although it is hard to explain the reason, we would prefer to suppose that an increase in symmetric cell divisions through altering cell cleavage orientations only occurs in the initial stage of the development of CSCC when the cell polarity still remains, by which cell proliferation is promoted. Along with progression, the cell polarity disap- pears and the cell cleavage orientation may no longer represent the cell division mode.

Different cell division modes were determined by the distinct distribution of cell fate determinants during mitosis in embryonic neurogenesis. Previous studies have revealed that Numb is inherited by both daughter cells during symmetric cell division, while Numb is inherited by only one daughter cell during asymmetric cell division no matter in Drosophila or mammals $[8,9]$. Numb can antagonize Notch signaling and thereby determine daughter cell fate [15-17]. It has been known that Notch signaling plays an important role in the maintenance of the proliferation/differentiation balance [18]. Alterations in Notch signaling have been implicated to be associated with tumorigenesis $[19,20]$. Our study discovered that Numb was expressed in the cervical squamous epithelia in all CSCC, CIN2/3, CIN1 cases and normal cervix. Numb staining appeared diffusely in the cytosol and membrane and had no asymmetric distribution. Numb expression had a tendency to be stronger from cervical normal epithelium through to CSCC.

It is known that human Numb has 4 splicing isforms, and distinct Numb isoforms have different subcellular localizations. It has been revealed that Numb-PRR ${ }^{\mathrm{L}}$ promotes cell proliferation of the cells whereas Numb$\mathrm{PRR}^{S}$ enhances differentiation [13]. In the present study, we found that the expression of Numb-PRR ${ }^{L}$ isoform mRNA was increased in CSCC. Our results suggest that increased expression of Numb-PRR ${ }^{\mathrm{L}}$ isoforms are probably the critical molecular events by which the cervical squamous cells obtain uncontrolled proliferative ability during their development into CSCC through CIN.

\section{Acknowledgement}

This project was supported by a grant from the Zhejiang Natural Science Foundation of China (Y204049, Y207162).

\begin{tabular}{|c|c|}
\hline References & $\begin{array}{l}1 \text { Horvitz HR, Herskowitz I: Mechanisms of } \\
\text { asymmetric cell division: two Bs or not two } \\
\text { Bs, that is the question. Cell 1992;68:237- } \\
255 \text {. } \\
2 \text { Huttner WB, Brand M: Asymmetric division } \\
\text { and polarity of neuroepithelial cells. Curr } \\
\text { Opin Neurobiol 1997;7:29-39. } \\
3 \text { Wodarz A, Huttner WB: Asymmetric cell di- } \\
\text { vision during neurogenesis in Drosophila } \\
\text { and vertebrates. Mech Dev 2003;120:1297- } \\
\text { 1309. }\end{array}$ \\
\hline
\end{tabular}


4 Caviness VS Jr, Takahashi T, Nowakowski RS: Numbers, time and neocortical neuronogenesis: a general developmental and evolutionary model. Trends Neurosci 1995;18: 379-383.

5 Chenn A, McConnell SK: Cleavage orientation and the asymmetric inheritance of Notch1 immunoreactivity in mammalian neurogenesis. Cell 1995;82:631-641.

-6 Cayouette M, Raff M: The orientation of cell division influences cell-fate choice in the developing mammalian retina. Development 2003;130:2329-2339.

7 Lin D, Edwards AS, Fawcett JP, et al: A mammalian PAR-3-PAR-6 complex implicated in Cdc42/Rac1 and aPKC signaling and cell polarity. Nat Cell Biol 2000;2:540-547.

-8 Shen Q, Zhong W, Jan YN, et al: Asymmetric Numb distribution is critical for asymmetric cell division of mouse cerebral cortical stem cells and neuroblasts. Development 2002; 129:4843-4853.
-9 Rhyu MS, Jan LY, Jan YN: Asymmetric distribution of numb protein during division of the sensory organ precursor cell confers distinct fates to daughter cells. Cell 1994;76: 477-491.

10 Cayouette M, Whitmore AV, Jeffery G, et al: Asymmetric segregation of Numb in retinal development and the influence of the pigmented epithelium. J Neurosci 2001;21: 5643-5651.

11 Bork P, Margolis B: A phosphotyrosine interaction domain. Cell 1995;80:693-694.

12 Verdi J, Schmandt R, Bashirullah A, et al: Mammalian Numb is an evolutionarily conserved signaling adapter protein that specifies cell fate. Curr Biol 1996;6:1134-1145.

$>13$ Verdi JM, Bashirullah A, Goldhawk DE, et al: Distinct human Numb isoforms regulate differentiation vs. proliferation in the neuronal lineage. Proc Natl Acad Sci USA 1999;96: 10472-10476.

14 Lechler T, Fuchs E: Asymmetric cell divisions promote stratification and differentiation of mammalian skin. Nature 2005;437: 275-280.

-15 Spana EP, Doe CQ: Numb antagonizes Notch signaling to specify sibling neuron cell fates. Neuron 1996;17:21-26.
16 Zhong W, Feder JN, Jiang MM, et al: Asymmetric localization of a mammalian numb homolog during mouse cortical neurogenesis. Neuron 1996;17:43-53.

-17 McGill MA, McGlade CJ: Mammalian numb proteins promote Notch1 receptor ubiquitination and degradation of the Notch1 intracellular domain. J Biol Chem 2003;278: 23196-23203.

18 Artavanis-Tsakonas S, Rand MD, Lake RJ: Notch signaling: cell fate control and signal integration in development. Science 1999; 284:770-776.

19 Gallahan D, Jhappan C, Robinson G, et al: Expression of a truncated Int 3 gene in developing secretory mammary epithelium specifically retards lobular differentiation resulting in tumorigenesis. Cancer Res 1996; 56:1775-1785.

20 Capobianco AJ, Zagouras P, Blaumueller $\mathrm{CM}$, et al: Neoplastic transformation by truncated alleles of human NOTCH1/TAN1 and NOTCH2. Mol Cell Biol 1997;17:62656273. 